\title{
Comparison of Healthcare Resource Utilization Between Patients Who Engaged or Did Not Engage With a Prescription Digital Therapeutic for Opioid Use Disorder
}

\author{
Fulton F Velez $\mathbb{D}^{1}$ \\ Sam Colman ${ }^{2}$ \\ Laura Kauffman ${ }^{2}$ \\ Charles Ruetsch (iD) ${ }^{3}$ \\ Kathryn Anastassopoulos $\mathbb{D}^{2}$ \\ Yuri A Maricich' \\ 'Pear Therapeutics, Inc., Boston, MA \\ USA; ${ }^{2}$ Covance Market Access, \\ Gaithersburg, MD, USA; ${ }^{3}$ Health \\ Analytics, Columbia, MD, USA
}

Background: A prescription digital therapeutic (PDT) (reSET- $\mathrm{O}^{\circledR}$ ) may expand access to behavioral treatment for patients with opioid use disorder (OUD) treated with buprenorphine, but long-term data on effectiveness are lacking.

Objective: To compare real-world healthcare resource utilization (HCRU) among patients who engaged with reSET-O and buprenorphine compared to similar patients in recovery treated with buprenorphine who did not fill their reSET-O script or engage with the PDT beyond week one. Methods: A retrospective analysis of facility and clinical service claims data was conducted in adults with PDT initiation and between 12 weeks and 9 months of continuous enrollment in a health plan after initiation. Patients who filled their prescription and engaged with the therapeutic were compared to patients who filled the prescription but did not engage beyond week one (NE), and patients who did not fill the prescription (NR) (the latter two groups combined into one group hereafter referred to as "non-engagers"). Comparisons were analyzed using a repeated-measures negative binomial model of encounters/procedures, adjusted for number of days in each period. Associated cost trends assessed using current Medicare reimbursement rates.

Results: A total of 444 patients redeemed a prescription and engaged with the PDT (mean age 37.5 years, $63.1 \%$ female, $84 \%$ Medicaid), and 64 patients did not engage with the PDT (mean age 39.5 years, 32.8\% female, $73.4 \%$ Medicaid). Total cost of hospital facility encounters was $\$ 2693$ for engaged patients vs $\$ 6130$ for non-engaged patients. Engaged patients had somewhat higher rates of certain clinician services. Total facility and clinician services costs for engaged vs non-engaged patients were $\$ 8733$ vs $\$ 11,441$, for a net cost savings over 9 months of $\$ 2708$ per patient who engaged with reSET-O.

Conclusion: Patients who engaged with an OUD-specific PDT had a net cost reduction for inpatient and outpatient services of $\$ 2708$ per patient over 9 months compared to patients who did not engage with the PDT, despite similar levels of buprenorphine adherence.

Keywords: community reinforcement approach, contingency management, healthcare resource utilization, opioid use disorder, OUD, prescription digital therapeutic, PDT, reSET-O

\section{Plain Language Summary}

Treating patients with opioid use disorder (OUD) reduces healthcare expenditures, yet roughly $80 \%$ of patients do not receive needed treatment. Prescription digital therapeutics (PDTs) are software-based treatments FDA-authorized to improve clinical outcomes for
Correspondence: Fulton F Velez

Pear Therape Floor 13, Boston, MA, 02109, USA

Email fulton.velez@peartherapeutics.com 
diseases and conditions. The reSET-O ${ }^{\circledR}$ PDT was approved in 2018 to help patients with OUD being concurrently treated with buprenorphine engage in treatment and reduce substance use, but to date there has been no long-term, real-world evaluation of how use of the PDT affects healthcare resource utilization (HCRU) patterns.

This study evaluated real-world use of healthcare resources in patients who engaged with reSET-O vs a control arm comprised of patients who were prescribed the PDT but who did not redeem their script (NR) or did not engage (NE) beyond week one (both groups of patients were being treated concurrently with buprenorphine). 444 patients redeemed a prescription and engaged with the therapeutic, and there were 64 patients in the $\mathrm{NR}+\mathrm{NE}$ group. Total cost of hospital facility encounters was $\$ 2693$ for engaged patients vs $\$ 6130$ for NR+NE patients. Engaged patients had somewhat higher rates of certain clinician services such as psychiatry services, case management, evaluation and management services, and pathology/drug testing. Total facility and clinician services costs for engaged vs NR+NE patients were $\$ 8733$ vs $\$ 11,441$, for a net cost savings over 9 months of $\$ 2708$ per patient who engaged with reSET-O.

The results of this study support the value of this PDT in the treatment of a patient population in need of more comprehensive support with recovery.

\section{Introduction}

Opioid use disorder (OUD) is a problematic pattern of opioid use with profound impacts on individuals, families, and society at large. ${ }^{1-6}$ The impact on direct healthcare costs in the US continues to grow and is currently estimated at approximately $\$ 90$ billion annually, which is primarily due to costs associated with hospital and emergency department (ED) encounters. ${ }^{3,7,8}$ The use of healthcare resources by patients with OUD may be increasing recently due to the COVID-19 pandemic, which has increased social isolation and has shifted care patterns to a range of remote or virtual modes. ${ }^{9,10}$ Opioid-related mortality rose sharply in the early months of the COVID-19 outbreak to its highest-ever recorded levels of daily deaths, exceeding 160 deaths/day in May 2020. ${ }^{11}$

Medications for opioid use disorder (MOUD) (ie, buprenorphine, methadone, or naltrexone), along with behavioral therapies and social supports are the preferred approaches for treating OUD. ${ }^{12,13}$ Buprenorphine, an opioid agonist that reduces withdrawal symptoms and cravings, has been shown to reduce the use of expensive healthcare services such as inpatient hospital stays and ED visits as well as lowering the total cost of care. ${ }^{7,12-18}$ MOUD has also been shown to reduce the risk of opioid overdose and to improve patient-centered outcomes such as mental health, employment, and residence in stable housing situations. ${ }^{12,14}$ Unfortunately, fewer than $20 \%$ of patients receive such treatment, ${ }^{19}$ patients face significant barriers to accessing MOUD, ${ }^{20}$ and fewer than $10 \%$ of patients receive adequate behavioral support, which contribute to reported high treatment dropout rates within 90 days (between $30 \%$ and 50\%), and may limit the effectiveness of MOUD and long-term recovery. ${ }^{21-23}$ As a result, a need exists to scale up the availability and provision of behavioral treatment and reduce waitlist times, a major reason for the call by the National Institute on Drug Abuse for development of digital versions of cognitive-behavioral therapy for addiction disorders. $^{24}$

reSET-O ${ }^{\circledR}$ was authorized by the FDA in 2018 for patients with OUD being treated with buprenorphine. It is an 84-day (12-week) prescription digital therapeutic $(\mathrm{PDT})^{25}$ that delivers an OUD-specific form of cognitive behavioral therapy (CBT) based on the community reinforcement approach (CRA). On-demand lessons delivered on smartphones or tablets via text, pre-recorded video, and audio increase patients' self-efficacy in avoiding illicit substances, and help to increase their ability to live a productive life free from opioids. Content retention and mastery is reinforced by fluency training (a series of simple repetitive quizzes at the end of each lesson) and by contingency management (eligibility for positive reinforcement messages or monetary digital gift cards) for engagement with lessons, up to 4 per week and for negative urine drug screens) (Figure 1).

In the clinical trials that formed the basis of FDA approval, $82 \%$ of OUD patients who received treatment with the PDT were retained in treatment vs $68 \%$ of patients receiving only treatment as usual (TAU) $(\mathrm{P}=$ 0.018 ), and the likelihood of abstinence during weeks 912 was $77.3 \%$ vs $62.1 \%$, respectively $(\mathrm{P}=0.011) .^{25}$

The current study seeks to evaluate real-world differences in healthcare resource utilization (HCRU) between patients who engaged, and patients who did not engage with the PDT over a period of 9 months following PDT treatment initiation.

\section{Methods}

This study was a real-world, retrospective analysis of facility and clinical service encounters in adults with OUD in treatment with buprenorphine MOUD (Figure 2). 


\section{PATIENT}

\section{CLINICIAN}

\section{Provides Intervention \\ - Cognitive Behavioral Therapy (CBT) Modules \\ - Fluency Training \\ - Contingency Management \\ - Craving and Trigger Assessment}

Improves Patient Access To Treatment
Provides Insights

- Real-World Engagement

- CBT Module Use

- Fluency Training

- Contingency Management

- Cravings and Triggers

- Urine Drug Screens and Appointments

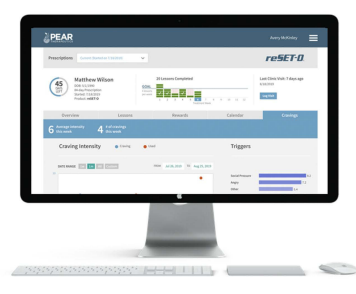

Improves Provider Insights To Treatment

Figure I Example of patient and clinician interfaces for reSET-O PDT.

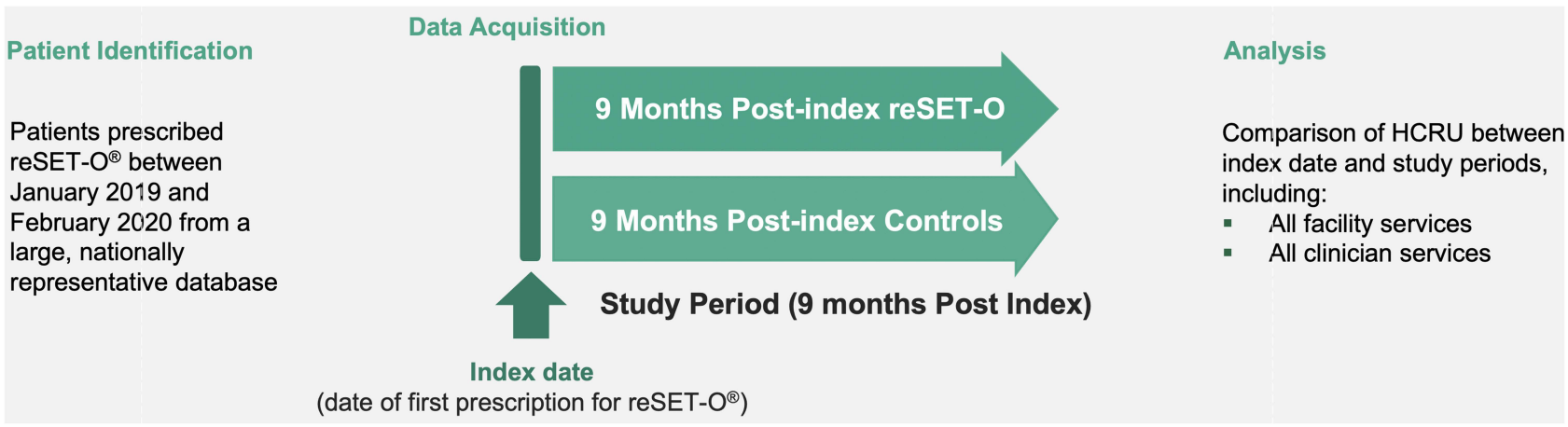

Figure 2 Study design.

\section{Patient Population}

The population analyzed was comprised of: 1) patients who received a prescription for reSET-O from a licensed clinician as part of their substance use disorder treatment (ie, these were patients treated in usual care settings, not recruited or otherwise incentivized to participate in a study), 2) had claims data available for analysis (as detailed below), and 3) had continuous enrollment in the medical/pharmacy plan for between 12 weeks and 9-month post-index period. Upon prescription, the PDT was activated (at no cost to the patient) and a claim was then submitted to the patient's health insurer. Patients were grouped by level of engagement with the PDT: patients who redeemed their prescription and engaged with the therapeutic after week 1 of PDT activation (engaged patients) vs patients who either redeemed the prescription but did not engage after week 1 or patients who did not redeem the prescription (non-engaged patients). The patients in both groups received concurrent buprenorphine therapy. No other exclusion/inclusion criteria were applied in order to maintain a broad sample of patients treated in usual care settings and who were considered by a treating clinician to be similarly suitable for a PDT prescription.

\section{Data Source}

Medical and pharmacy claims from the HealthVerity PrivateSource20 (PS20) database between January 2019 and February 2020 (study period) were the source of data for this study. The PS20 database includes medical and pharmacy claims for about 70 million commercial, 60 million Medicaid, and 15 million Medicare enrollees distributed across 150 payers since 2015 . This study received a waiver of authorization for the use and disclosure of protected health information (PHI) and a determination of exempt status under 45 CFR $\S$ 46.104(d)(4) from the Western Institutional Review Board on April 2, 2020. 


\section{Study Measures}

Patient demographics including age, sex, and payer type were analyzed. Post-index period claims were aggregated as facility claims or clinical service claims to organize patients' use of healthcare resources. Facility encounters included all-cause inpatient stays, and use of intensive care unit (ICU), emergency department (ED), partial hospitalizations (PH), and surgical outpatient department (SOD) facilities. Assessed clinical service encounters included all-cause Current Procedure Terminology (CPT) codes identified from clinician claims such as evaluation and management codes, medical codes (eg, cardiovascular, psychiatry, neurology), pathology and laboratory, and rehabilitative services. Adherence to buprenorphine was analyzed using the medication possession ratio (MPR) on filled prescriptions in the post-index period for the engaged and non-engaged cohorts.

\section{Analyses}

Healthcare costs were calculated by applying Medicare costs to the incidence rate observed for the different healthcare resource utilization categories. This provides an accurate, albeit conservative, estimate of healthcare costs borne by the system. ${ }^{26}$ Cost differences between engaged and non-engaged patients were calculated. MPR was calculated as the total days' supply of buprenorphine within the post-index period divided by the total number of days in the post-index period. ${ }^{27}$

\section{Results}

\section{Patient Demographic and Clinical Characteristics}

444 patients redeemed a prescription for reSET-O and engaged with the therapeutic (mean age 37.5 years, $63.1 \%$ female, $84 \%$ Medicaid), and 64 patients either did not redeem their prescription or redeemed the prescription but did not engage with the therapeutic (mean age 39.5 years, $32.8 \%$ female, $73.4 \%$ Medicaid). Evidence of claims for buprenorphine in the 9-month pre-index period was available for $78 \%$ of engaged patients (345 of 444) vs $67 \%$ of non-engaged patients (43 of 64 ). In the 9-month pre-index period the per-patient rate of psychiatric consultations was 9.11 in engaged patients vs 5.14 in nonengaged patients.

\section{Facility Encounters Comparison}

The incidence rate for unique hospital encounters in the 9-month post-index period was 0.723 for engaged patients vs 1.102 for non-engaged patients, with the largest differences observed for inpatient stays (engaged: 0.139 vs nonengaged: 0.256$)$, ICU stays (0.024 vs 0.054$)$, and partial hospitalizations $(0.025$ vs 0.288$)$. Detailed results are shown in Table 1.

\section{Clinical Service Encounters Comparison}

Engaged patients had somewhat higher rates of certain clinician services such as psychiatry services, case management, evaluation and management services, and pathology/drug testing. Detailed results are shown in Table 2.

\section{Total Cost Comparison}

Total cost of facility encounters was \$2693 for engaged patients vs $\$ 6130$ for non-engaged patients, while total per-patient cost for clinician services was $\$ 6040$ for engaged patients vs $\$ 5311$ for non-engaged patients (Table 3). The lower facility encounter costs among engaged patients outweighed their slightly greater clinician services costs vs non-engaged patients resulting in lower overall costs of $\$ 8733$ for engaged vs $\$ 11,441$ for non-

Table I Incidence Rates for Hospital Encounters in 9-Month Post-Index Period

\begin{tabular}{|l|l|l|}
\hline Resource & $\begin{array}{l}\text { Incidence (95\% CI) Among Engaged } \\
\text { Patients (N=444) }\end{array}$ & $\begin{array}{l}\text { Incidence (95\% CI) Among Non-Engaged } \\
\text { Patients (N=64) }\end{array}$ \\
\hline Unique hospital encounters & $0.723(0.569-0.918)$ & $1.102(0.594-2.045)$ \\
Inpatient stays & $0.139(0.097-0.20 I)$ & $0.256(0.119-0.549)$ \\
ICU stays & $0.024(0.010-0.055)$ & $0.054(0.014-0.214)$ \\
Partial hospitalizations & $0.025(0.006-0.102)$ & $0.288(0.049-1.707)$ \\
ED visits-not admitted & $0.549(0.419-0.719)$ & $0.542(0.304-0.967)$ \\
HOPD visits-not admitted & $0.007(0.001-0.046)$ & $0.000(0.000-0.047)$ \\
\hline
\end{tabular}

Abbreviations: $\mathrm{Cl}$, confidence interval; ED, emergency department; ICU, intensive care unit; HOPD, hospital outpatient department. 
Table 2 Incidence Rates for Physician Services in 9-Month Post-Index Period

\begin{tabular}{|l|l|l|}
\hline Procedure & $\begin{array}{l}\text { Incidence (95\% CI) Among Engaged } \\
\text { Patients } \mathbf{( N = 4 4 4 )}\end{array}$ & $\begin{array}{l}\text { Incidence (95\% CI) Among Non-Engaged } \\
\text { Patients (N=64) }\end{array}$ \\
\hline Dental & $0.460(0.357-0.592)$ & $0.425(0.193-0.936)$ \\
\hline Case management services & $11.068(7.990-15.333)$ & $6.201(1.727-22.267)$ \\
\hline Consultation services & $0.128(0.076-0.215)$ & $0.100(0.040-0.253)$ \\
\hline E\&M hospital inpatient services & $0.703(0.404-1.224)$ & $1.365(0.567-3.284)$ \\
\hline E\&M office/other outpatient services & $17.495(16.274-18.807)$ & $14.460(11.608-18.012)$ \\
\hline Medicine: Cardiovascular & $0.367(0.021-0.081)$ & $0.361(0.175-0.747)$ \\
\hline Medicine: Central nervous system assessments & $0.041(0.021-0.081)$ & $0.023(0.003-0.166)$ \\
\hline Medicine: Neurology and neuromuscular & $0.087(0.054-0.141)$ & $0.048(0.012-0.187)$ \\
\hline procedures & & $6.269(4.012-9.795)$ \\
\hline Medicine: Psychiatry & $9.130(8.034-10.375)$ & $0.045(0.006-0.312)$ \\
\hline Medicine: Pulmonary & $0.117(0.074-0.185)$ & $10.139(7.755-13.256)$ \\
\hline Drug testing & $13.424(12.211-14.757$ & $6.091(2.140-17.34 I)$ \\
\hline Alcohol and substance rehabilitative services & $2.048(1.282-3.271)$ & $1.088(0.655-1.806)$ \\
\hline Surgery & $1.110(0.928-1.328)$ & \\
\hline
\end{tabular}

Abbreviations: $\mathrm{Cl}$, confidence interval; E\&M, evaluation and management.

engaged patients, for an overall cost reduction of $\$ 2708$ per patient over 9 months for engaged patients.

\section{Buprenorphine Adherence Patterns}

Adherence to buprenorphine therapy rose among engaged patients (pre-index mean MPR $=0.69$; post-index mean MPR $=0.80, \mathrm{P}<0.0001)$ as well as among the nonengaged patients although the pre/post difference among non-engagers did not reach statistical significance (preindex mean $\mathrm{MPR}=0.62$; post-index mean $\mathrm{MPR}=0.79$; $\mathrm{P}=0.0597)$.

\section{Discussion}

This real-world claims data analysis of patients with OUD on buprenorphine MOUD who either did, or did not, engage with a PDT showed substantially lower incidence rates of hospital encounters among engaged patients compared to non-engaged patients, especially in inpatient stays and ICU stays. There were somewhat higher rates of certain physician services among the engaged patients, for example for case management services, evaluation and management outpatient services, neurology and neuromuscular procedures, psychiatry services, and drug testing. In the case of psychiatric consultations, the rate was comparable to that observed in the pre-index period for engaged patients, but non-engaged patients had an increase of $22 \%$ vs the pre-index period. These higher rates of some physician services observed in patients who engaged with reSET-O, however, were more than offset by lower rates of the more expensive hospital services.

The net cost savings over 9 months of $\$ 2708$ per patient who engaged with reSET-O that we observed in this evaluation is consistent with previously published evaluations of the economic impacts of reSET-O using modeling

Table 3 Cost Comparisons, Engaged vs Non-Engaged Patients

\begin{tabular}{|l|c|c|c|}
\hline & Cost/Engaged Patient (n=444) & Cost/Non-Engaged Patient (n=64) & Difference \\
\hline Inpatient facility services & $\$ 2,693$ & $\$ 6,130$ & $(\$ 3,437)$ \\
\hline Inpatient clinician services & $\$ 6,040$ & $\$ 5,311$ & $\$ 729$ \\
\hline Net cost difference & & & $\$ 2,708$ \\
\hline
\end{tabular}


approaches, which showed the potential for reSET-O to produce overall reductions in healthcare costs net of product cost. $^{28,29}$ The present study confirms those observations, as the $\$ 2708$ cost difference observed between controls and reSET-O patients exceeds the current discounted price of a 12-week prescription for the therapeutic $(\$ 1440)$.

Given that the cost assumptions used in this study are for the most part conservative, a more comprehensive cost analysis incorporating additional cost scenarios, as well as changes in efficiency of care (ie, impact of reduced perpatient face-to-face clinician time through PDT-enabled CBT delivery) should be conducted to determine additional cost impacts.

Recovery and abstinence are long-term goals facilitated by retention in therapy to help reduce excess hospitalizations and ED visits through continued patient reinforcement and the reduction in exposure to opioids and the risk of accidental overdose.

These results are specific to an FDA-authorized PDT and may not be generalizable to a non-FDA-authorized digital therapeutic. FDA authorization is important in ensuring safety, efficacy, and quality of software as a medical device. ${ }^{30-32}$

\section{Limitations}

As with any healthcare claims-based analysis, there are potential limitations and mitigation factors that should be noted. For instance, claims databases are structured for administrative purposes, not research purposes, and may contain coding errors or missing information. Claims databases also do not provide data about severity of disease or about whether specific health conditions are associated with OUD or not (eg, stroke associated with an accidental overdose vs no association with an overdose event), limiting the conclusions that can be drawn about the PDT intervention. The results reported indicate an association between outcomes and exposure to the PDT, not a causal relationship, which will require larger studies that control for confounding. However, claims databases do provide a broad view into the burden to a healthcare system in patients with a particular condition, or undergoing treatment with a specific healthcare technology.

The sample size of the non-engaged patient population was relatively small compared to the engaged patient population, which calls for caution in interpreting these results. Nevertheless, there was evidence of increased adherence to buprenorphine in non-engaged patients that was similar to the engaged patients, and the results are therefore suggestive of the persistent impact of behavioral support provided by an OUD-specific PDT up to nine months post-treatment initiation (or six months following treatment completion).

Some degree of selection bias may be present in these samples as well. For example, it is possible that nonengaged patients may be at an earlier (and thus more vulnerable) phase of their recovery, which could partially explain the higher healthcare utilization observed. This is mitigated by the fact that all the patients in this analysis were prescribed the PDT by their treating clinician, potentially a proxy marker of being at a similar readiness to change stage in the recovery journey, which is further supported by the increased buprenorphine adherence in the non-engaged control arm, to levels comparable to the PDT arm. It should also be noted that non-engaged patients were more likely to be male, although it is not currently known to what extent this might impact the results. Data about the severity of patients' OUD or their stage of recovery was not available for this analysis; such data would allow a more comprehensive exploration of their impact on healthcare resource utilization patterns alongside intervention with a PDT.

\section{Conclusions}

In this real-world observational study of patients prescribed an OUD-specific PDT using concurrent buprenorphine therapy, engagement with the PDT was associated with a net cost reduction for inpatient and outpatient services of $\$ 2708$ per patient over a 9-month treatment period. The results of this study support the value of this PDT in the treatment of a patient population in need of more comprehensive support with recovery.

\section{Summary}

Why carry out this study?

- Clinical trial data support the efficacy of a prescription digital therapeutic for patients with opioid use disorder (OUD), but real-world healthcare resource utilization (HCRU) data have been lacking.

- This study of 444 patients with OUD on buprenorphine therapy compared those who engaged with a PDT and those who did not and evaluated HCRU outcomes and associated costs.

What was learned from the study? 
- Total facility and clinician services costs for engaged vs. non-engaged patients were $\$ 8,733$ vs. $\$ 11,441$, for a net cost savings over 9 months of $\$ 2,708$ per patient who engaged with the PDT.

- The results provide evidence of robust cost reductions with a PDT in the treatment of a patient population in need of more comprehensive support with recovery.

\section{Acknowledgments}

The authors would like to thank Stephen Braun for assistance with writing support and critical review of the manuscript. These analyses were funded entirely by Pear Therapeutics, Inc., which developed and markets reSET-O.

\section{Author Contributions}

All authors made a significant contribution to the work reported, whether that is in the conception, study design, execution, acquisition of data, analysis and interpretation, or in all these areas; took part in drafting, revising or critically reviewing the article; gave final approval of the version to be published; have agreed on the journal to which the article has been submitted; and agree to be accountable for all aspects of the work.

\section{Disclosure}

Dr Velez and Dr Maricich are employees of Pear Therapeutics and have no additional conflicts of interest to disclose. Ms Kauffman reports personal fees from Pear Therapeutics, Inc., during the conduct of the study. Dr Ruetsch reports personal fees from Pear Therapeutics and Indivior, outside the submitted work. Ms Anastassopoulos is an employee of Labcorp which was contracted to conduct the study. The authors report no other conflicts of interest in this work.

\section{References}

1. Jalal H, Buchanich JM, Roberts MS, Balmert LC, Zhang K, Burke DS. Changing dynamics of the drug overdose epidemic in the United States from 1979 through 2016. Science. 2018;361:6408. doi:10.1126/ science.aau 1184

2. Hagemeier NE. Introduction to the opioid epidemic: the economic burden on the healthcare system and impact on quality of life. Am J Manag Care. 2018;24(10 Suppl):S200-S206.

3. Florence CS, Zhou C, Luo F, Xu L. The economic burden of prescription opioid overdose, abuse, and dependence in the United States, 2013. Med Care. 2016;54(10):901-906. doi:10.1097/MLR.000 0000000000625

4. Ray GT, Mertens JR, Weisner C. The excess medical cost and health problems of family members of persons diagnosed with alcohol or drug problems. Med Care. 2007;45(2):116-122. doi:10.1097/01. mlr.0000241109.55054.04
5. Kirson NY, Scarpati LM, Enloe CJ, Dincer AP, Birnbaum HG, Mayne TJ. The economic burden of opioid abuse: updated findings. J Manag Care Spec Pharm. 2017;23(4):427-445.

6. Klein AA, Anker JJ. Computer-based recovery support for patients receiving residential treatment for alcohol/drug dependence: relationship between program use and outcomes. Telemed $J E$ Health. 2012;19(2):104-109. doi:10.1089/tmj.2012.0107

7. The Council of Economic Advisers. The underestimated cost of the opioid crisis; 2017. Available from: https://www.whitehouse.gov/ briefings-statements/cea-report-underestimated-cost-opioid-crisis/. Accessed October 19, 2021.

8. Murphy SM. The cost of opioid use disorder and the value of aversion. Drug Alcohol Depend. 2020;217:108382. doi:10.1016/j. drugalcdep.2020.108382

9. Becker WC, Fiellin DA. When epidemics collide: coronavirus disease 2019 (COVID-19) and the opioid crisis. Ann Intern Med. 2020;173(1):59-60. doi:10.7326/M20-1210

10. The Opioid Response Network. The opioid epidemic and COVID-19: how social determinants of health create differential impacts; 2020. Available from: https://opioidresponsenetwork.org/documents/SDH OUD-COVID-19.pdf. Accessed July 22, 2020.

11. American Medical Association (AMA). Issue brief: reports of increases in opioid-related overdose and other concerns during COVID pandemic; 2020. Available from: https://www.ama-assn.org/ system/files/2020-07/issue-brief-increases-in-opioid-related-over dose.pdf. Accessed July 22, 2020.

12. Wakeman SE, Larochelle MR, Ameli O, et al. Comparative effectiveness of different treatment pathways for opioid use disorder. JAMA Network Open. 2020;3(2):e1920622-e1920622. doi:10.1001/ jamanetworkopen.2019.20622

13. Boyett B. The individualized treatment of opioid use disorder. J Manag Care Spec Pharm. 2019;25(6):634-636.

14. U.S. Department of health \& human services. In: Facing Addiction in America - the Surgeon General's Spotlight on Opioids. Washington, DC; 2018.

15. Ling W, Nadipelli VR, Solem CT, et al. Effects of monthly buprenorphine extended-release injections on patient-centered outcomes: a long-term study. J Subst Abuse Treat. 2020;110:1-8. doi:10.1016/j. jsat.2019.11.004

16. Ruetsch C, Tkacz J, Nadipelli VR, et al. Heterogeneity of nonadherent buprenorphine patients: subgroup characteristics and outcomes. Am J Manag Care. 2017;23(6):e172-e179.

17. Lynch FL, McCarty D, Mertens J, et al. Costs of care for persons with opioid dependence in commercial integrated health systems. Addict Sci Clin Pract. 2014;9(1):16. doi:10.1186/1940-0640-9-16

18. Ronquest NA, Willson TM, Montejano LB, Nadipelli VR, Wollschlaeger BA. Relationship between buprenorphine adherence and relapse, health care utilization and costs in privately and publicly insured patients with opioid use disorder. Subst Abuse Rehabil. 2018;9:59-78. doi:10.2147/SAR.S150253

19. Substance Abuse and Mental Health Services Administration. National Survey of Drug Use and Health (NSDUH) releases; 2019. Available from: https://www.samhsa.gov/data/release/2019-nationalsurvey-drug-use-and-health-nsduh-releases. Accessed September 29, 2020.

20. Hall NY, Le L, Majmudar I, Mihalopoulos C. Barriers to accessing opioid substitution treatment for opioid use disorder: a systematic review from the client perspective. Drug Alcohol Depend. 2021;221:108651. doi:10.1016/j.drugalcdep.2021.108651

21. Shulman M, Weiss R, Rotrosen J, Novo P, Costello E, Nunes EV. Prior national drug abuse treatment Clinical Trials Network (CTN) opioid use disorder trials as background and rationale for NIDA CTN-0100 "optimizing retention, duration and discontinuation strategies for opioid use disorder pharmacotherapy (RDD)". Addict Sci Clin Pract. 2021;16(1):15. doi:10.1186/s13722-021-00223-z 
22. Bentzley BS, Barth KS, Back SE, Book SW. Discontinuation of buprenorphine maintenance therapy: perspectives and outcomes. J Subst Abuse Treat. 2015;52:48-57. doi:10.1016/j.jsat.2014.12.011

23. Dutra L, Stathopoulou G, Basden SL, Leyro TM, Powers MB, Otto MW. A meta-analytic review of psychosocial interventions for substance use disorders. Am J Psychiatry. 2008;165(2):179-187. doi:10.1176/appi.ajp.2007.06111851

24. National Institute on Drug Abuse. Principles of drug addiction treatment: a research-based guide (Third edition): drug addiction treatment in the United States. Available from: https:/www.drugabuse. gov/publications/principles-drug-addiction-treatment-research-basedguide-third-edition/drug-addiction-treatment-in-united-states. Accessed April 24, 2021.

25. Pear Therapeutics Inc. reSET-O [clinician directions for use]; 2017.

26. Centers for Medicare and Medicaid Services (CMS). Medicare hospital outpatient prospective payment system addendum A, April 2020 file; 2020. Available from: https://www.cms.gov/Medicare/MedicareFee-For-Service-Payment/HospitalOutpatientPPS/Addendum-A-andAddendum-B-Updates. Accessed July 28, 2020.

27. Cramer JA, Roy A, Burrell A, et al. Medication compliance and persistence: terminology and definitions. Value Health. 2008;11 (1):44-47. doi:10.1111/j.1524-4733.2007.00213.x
28. Velez FF, Colman S, Kauffman L, Ruetsch C, Anastassopoulos K. Real-world reduction in healthcare resource utilization following treatment of opioid use disorder with reSET-O, a novel prescription digital therapeutic. Expert Rev Pharmacoecon Outcomes Res. 2020;21:69-76. PMID: 33146558.

29. Velez FF, Colman S, Kauffman L, Anastassopoulos K, Murphy S, Maricich Y. Real-world changes in US health system hospital-based services following treatment with a prescription digital therapeutic for opioid use disorder. Hosp Pract. 2021;1-7. doi:10.1080/ 21548331.2021.1956256

30. Khadjesari Z, Brown T, Naughton F. Regulation and accreditation of addictive behaviour applications-navigating the landscape. Addiction. 2021. doi:10.1111/add.15484

31. Tarricone R, Petracca F, Ciani O, Cucciniello M. Distinguishing features in the assessment of mHealth apps. Expert Rev Pharmacoecon Outcomes Res. 2021;1-6. doi:10.1080/ 14737167.2021.1891883

32. Ambrose M. Ensuring public trust in digital therapeutics: a pharmacopeial perspective. J Manag Care Spec Pharm. 2021;27 (4):533-535.
ClinicoEconomics and Outcomes Research

\section{Publish your work in this journal}

ClinicoEconomics and Outcomes Research is an international, peerreviewed open-access journal focusing on Health Technology Assessment, Pharmacoeconomics and Outcomes Research in the areas of diagnosis, medical devices, and clinical, surgical and pharmacological intervention. The economic impact of health policy and health systems

\section{Dovepress}

organization also constitute important areas of coverage. The manuscript management system is completely online and includes a very quick and fair peer-review system, which is all easy to use. Visit http://www.dovepress.com/testimonials.php to read real quotes from published authors. 